\title{
Stretching of the mechanical-geometric model and two common nonlinear elastic solids
}

\author{
Daniil Azarov ${ }^{1, *}$ \\ ${ }^{1}$ Don State Technical University, 1, Gagarina sq., 344003, Rostov-on-Don, Russia
}

\begin{abstract}
The variety of hyperelastic materials and the design of new modifications and technical applications requires the development of a description of nonlinear deformation properties. The most commonly used constitutive relations of the Mooney-Rivlin and Yeoh models are based on polynomial decompositions. Mechanical-geometric modeling (hereinafter MGM) is a new way of constructing constitutive relations and strain energy densities within the nonlinear theory of elasticity. In this paper, a comparison of the deformation behavior of MGM with the traditional Mooney-Rivlin and Yeoh models was carried out. Comparative analysis is accompanied by diagrams for uniaxial and biaxial stretching. The effectiveness of the new model was proved.
\end{abstract}

\section{Introduction}

A huge variety of various materials that undergoes the large deformations is used in modern manufacturing and engineering. In recent decades, standard structural materials such as steel, alloys and wood have been actively supplanted by new ones. They are various polymers, composites, natural and artificial rubbers. Their elongation deformations can be many times larger than the original sizes of the samples, thus they are called hyperelastic materials. In addition to artificial materials, many natural appear to be highly elastic: vessel walls, ligaments, etc. $[1,2]$.

The linear theory of elasticity fails to describe the properties of such materials, as their reactions to external loads are significantly non-linear. The problem of constructing constitutive relations for such materials is also relevant due to the appearance of materials with complex structures and physical properties [3-8].

When constructing the constitutive relations of the nonlinear theory of elasticity, the method of decomposing the elastic strain energy function into a power series in invariants of one of the strain tensors, followed by retaining values of a certain order of smallness in this decomposition, remains one of the main and most often used method. Many common strain energy densities of the nonlinear theory of elasticity, such as Mooney-Rivlin (see Mooney M. A theory of large elastic deformation), neo-Hookean, Yeoh (see Yeoh O. H. Some forms of the strain energy function for rubber), Biderman and others were obtained through this procedure. A positive feature of this approach is its simplicity. But at the same time, the choice of the order of smallness of the discarded members of the series, as well as

\footnotetext{
*Corresponding author: danila_az@mail.ru
} 
the even-odd function property of the strain energy density obtained must be proven properly. The resulting expressions require justification for potential applications.

One of the first potentials based on the decomposition of specific strain energy density not by invariants of the Cauchy-Green tensor, but by the principal strains (elongations) $\lambda_{1}, \lambda_{2}, \lambda_{3}$ was the Ogden potential (see Ogden R. W. Large Deformation Isotropic Elasticity) with the specific strain energy density

$$
W=\sum_{i=1}^{n} \frac{2 \mu}{\alpha_{i}^{2}}\left(\lambda_{1}^{\alpha_{i}}+\lambda_{2}^{\alpha_{i}}+\lambda_{3}^{\alpha_{i}}-3\right)
$$

here $^{\mu, \alpha}$ are material constants of the model.

The great advantage of this form of the energy function is that the principal elongations are values that are easily measurable. The Ogden potential has recently been used increasingly, in particular to describe the properties of biological tissues.

Another approach, based on statistical physics and some assumptions about the structure of the material, leads to the Gent strain energy density (see Gent A. N. A new constitutive relation for rubber.)

$$
W=-\frac{\mu J_{m}}{2} \ln \left(1-\frac{I_{1}-3}{J_{m}}\right)
$$

here $I_{1}$ is the first invariant of the Cauchy-Green strain measure $\boldsymbol{G}_{:} I_{1}=\lambda_{1}{ }^{2}+\lambda_{2}^{2}+\lambda_{3}^{2}$ and $\mu, J_{m}$ are material constants of the model, or Arruda-Boyce strain energy density (see Arruda E. M., Boyce M. C. A three-dimensional model for the large stretch behavior of rubber elastic materials):

$$
W=N k_{B} \theta \sqrt{n}\left(\beta \lambda_{c h}-\sqrt{n} \ln \left(\frac{\operatorname{sh} \beta}{\beta}\right)\right)
$$

$\lambda_{\text {here }}=\sqrt{\frac{I_{1}}{3}}$ and $N, k_{B}, \theta, n, \beta$ are parameters of the model.

Despite their rather complex shape, these forms of energy have recently also been used quite widely due to their good approximation capabilities of the s-curve diagram of elastomer's stretch.

The parameters of most models are obtained through experimental data of uniaxial stretching and there are very rare estimations (or analyses) of the behavior of the solid's model in conditions of different stress-strain types [9].

All materials described above are incompressible in the original statement. When describing the behavior of elastomers (hyperelastic materials), this property of incompressibility is often an important simplifying factor. The application of the incompressibility hypothesis to such materials can be justified by the fact that this hypothesis allows one to obtain analytical solutions to a large class of problems. However, real materials are not ideally incompressible, which leads to the need to introduce certain terms into their models that make them compressible. Usually this is a term of the kind: $D(J-1)^{2}$. Here $D$ is a factor varying from 0 (i.e. fully incompressible material) up to 1 , and $J=\sqrt{I_{3}}, I_{3}=\lambda_{1}{ }^{2} \lambda_{2}{ }^{2} \lambda_{3}{ }^{2}$ is the third invariant of the Cauchy-Green strain measure $\boldsymbol{G}$. This further complicates the mathematical setting of problems of the nonlinear theory of elasticity. 
An extensive overview of the contemporary strain energy densities of nonlinear solids is given in [10]

\section{Mechanical-geometric models}

The method of mechanical-geometric modeling (MGM) is a new method of obtaining constitutive relations and strain energy densities for nonlinear solids. The main idea of this method is to describe the deformation of the elementary volume of a solid similarly to the deformation of some mechanical structure (or 3D truss consisting of rods), the model is "as if inscribed" into this elementary volume.

In this spatial truss, it's rods work for stretching-compression without loss of stability. These rods are called model's bonds.

Model bonds are connected to each other at model joints. In the following article, the joints are hinged connections that do not transmit torque.

The initial geometry of the model bonds determines the type of anisotropy the model possesses. Symmetries of the model generate symmetries of the modeled medium. So, if you specify different dimensions of the model for three dimensions, then the modeled environment will be orthotropic. Bellow in this paper, the model before deformation is a cube, which accordingly leads to an isotropic model. Fig. 1 shows two possible geometry types of models' bonds.
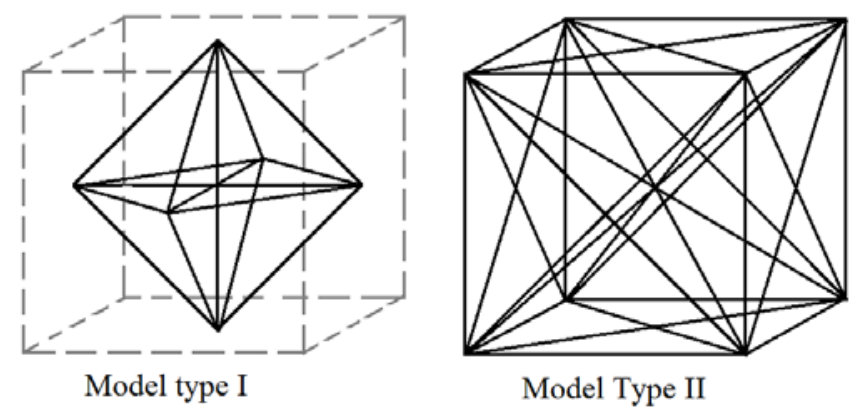

Fig. 1. Examples of cubic MGM model geometry.

When external forces are applied to the joints of the model's bonds, the corresponding forces (internal forces of the model) arise within these bonds. All model bonds are deformed (with corresponding elongations $\Delta_{i}$, where $i$ is the index of the bond) and the spatial dimensions and angles of the truss are changed.

Mechanical properties such as elasticity, viscosity, plasticity, or their combinations are attributed to model bonds. To describe high elasticity, it is more suitable to consider rods as compliant springs. In this paper, models are considered only with elastic bonds characterized by a linear dependence $F_{i}=k_{i} \Delta_{i}$ of force $F_{i}$ on elongation $\Delta_{i}$, and rigidities of bonds $k_{a}, k_{l}$ and $k_{d}$ here and bellow are constants of the model.

Based on the model's bonds deformation analysis, when external forces are applied to joints (with hinged connections) during triaxial deformation, the dependencies of changes in relative dimensions from forces in three directions were obtained.

Passing from forces to face stress characteristics, model constitutive relations in the principal axes were then derived. The corresponding equations describe the dependencies of the principal (axial) engineering stresses $\sigma_{1}, \sigma_{2}, \sigma_{3}$ on the principal (axial) ratios of 
extensions $\lambda_{1}, \lambda_{2}, \lambda_{3}$. From this point of view, the MG model is similar to the Ogden model. Principal ratios of extensions $\lambda_{i}$ can be defined through absolute extensions $\Delta_{i}$.

Basing on the total differential of the function, the specific potential strain energy function is reconstructed according to the known procedure. Thus, for MGM, expressions of the specific strain energy densities of isotropic solid were obtained for the two models shown in Fig. 1:

$$
\begin{aligned}
& W_{I}=\frac{1}{8}\left[\left(k_{a}+4 k_{l}\right)\left(\lambda_{1}^{2}+\lambda_{2}^{2}+\lambda_{3}^{2}\right)-\right. \\
& -4 \sqrt{2} k_{l}\left(\sqrt{\lambda_{1}^{2}+\lambda_{2}^{2}}+\sqrt{\lambda_{1}^{2}+\lambda_{3}^{2}}+\sqrt{\lambda_{2}^{2}+\lambda_{3}^{2}}\right)- \\
& \left.-2 k_{a}\left(\lambda_{1}+\lambda_{2}+\lambda_{3}\right)\right]+\frac{3}{8}\left(k_{a}+4 k_{l}\right) \\
& W_{I I}=2\left(k_{a}+2 k_{l}+k_{d}\right)\left(\lambda_{1}^{2}+\lambda_{2}^{2}+\lambda_{3}^{2}\right)-4 \sqrt{3} k_{d} \sqrt{\lambda_{1}^{2}+\lambda_{2}^{2}+\lambda_{3}^{2}}- \\
& -4 \sqrt{2} k_{l}\left(\sqrt{\lambda_{1}^{2}+\lambda_{2}^{2}}+\sqrt{\lambda_{1}^{2}+\lambda_{3}^{2}}+\sqrt{\lambda_{2}^{2}+\lambda_{3}^{2}}\right)- \\
& -4 k_{a}\left(\lambda_{1}+\lambda_{2}+\lambda_{3}\right)+6\left(k_{a}+2 k_{l}+k_{d}\right)
\end{aligned}
$$

here, $k_{a}, k_{l}$ and $k_{d}$ are model parameters, that have a clear mechanical meaning of bonds' stiffnesses. Further in this paper the model of type II, being more general, will be considered.

\section{Models choice for comparison}

Fig. 2 shows the diagrams of uniaxial stretch for some of the most common models of hyperelastic materials in the nonlinear theory of elasticity.

One of the most widely used Mooney-Rivlin model has the following specific strain energy formula:

$$
W=C_{10}\left(I_{1}-3\right)+C_{01}\left(I_{2}-3\right)
$$

here $C_{10}, C_{01}$ are the material constants, $I_{1}, I_{2}$ are the first and second invariants of the Cauchy-Green strain measure $\boldsymbol{G}: I_{1}=\lambda_{1}{ }^{2}+\lambda_{2}{ }^{2}+\lambda_{3}{ }^{2}, I_{2}=\lambda_{1}{ }^{2} \lambda_{2}{ }^{2}+\lambda_{2}{ }^{2} \lambda_{3}{ }^{2}+\lambda_{3}{ }^{2} \lambda_{1}{ }^{2}$.

Although the Mooney-Rivlin potential cannot adequately describe a typical s-shaped stretch diagram of hyperelastic materials, the frequency of its choice as a model material for natural and artificial rubbers is explained both by the simplicity of the formula itself and by the rather large amount of data on its material constants determined experimentally.

The Yeoh model with specific strain energy density

$$
W=C_{10}\left(I_{1}-3\right)+C_{20}\left(I_{1}-3\right)^{2}+C_{30}\left(I_{1}-3\right)^{3}
$$

is also widely used. Here $C_{10}, C_{20}, C_{30}$ - are the material constants.

One of its advantages is that it can describe an s-curve of rubber's stretch diagram. However, the amount of data on material parameters is much smaller than for the MooneyRivlin model. 


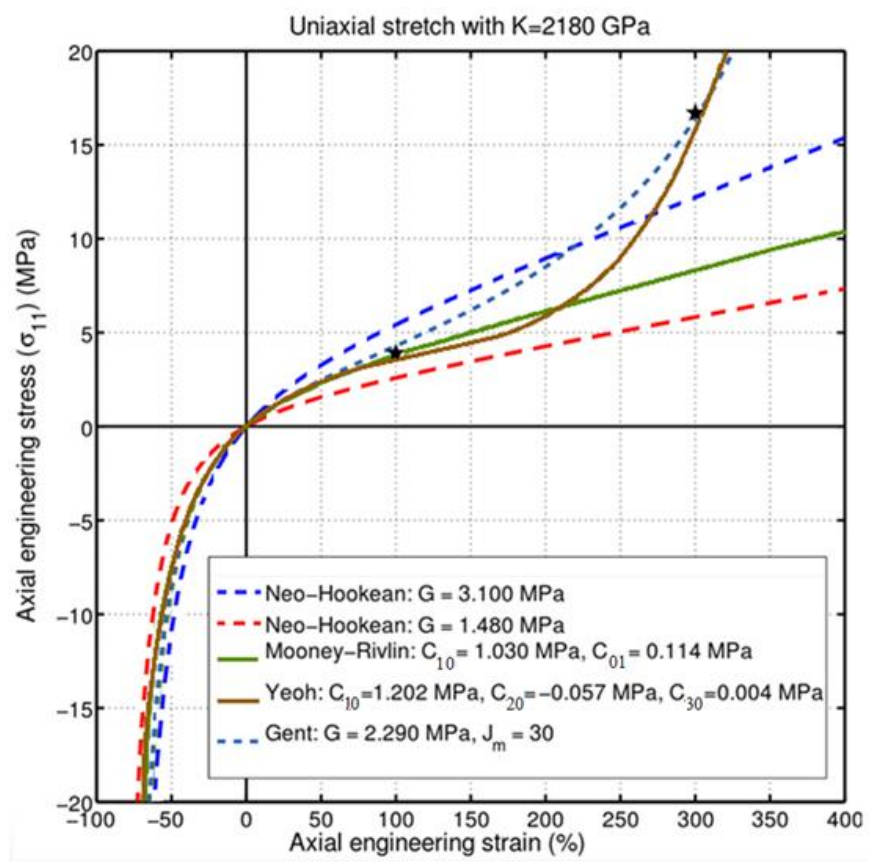

Fig. 2. Common models' uniaxial stretch diagrams:

https://en.wikipedia.org/wiki/Hyperelastic_material.

Both models, as shown in formulae (3) and (4), are typical polynomial models. Comparison with (1) and (2) shows the fundamental difference between MGM and traditional dependencies: neither of the models proposed up to this time had in the expression for the specific strain energy density square roots from the pairwise sums of squares of the main extensions or their triple sum of squares.

\section{Comparative models' analysis}

The calculations using the constitutive relations of two models (Mooney-Rivlin and Yeoh) were used as initial "as if experimental" data for identification of the parameters of the MG model in different stress-strain states.

The parameters of the MG model were determined by two data types:

1) the diagram of uniaxial stretching,

2) the diagram of equibiaxial stretching.

It should be noted that MGM is initially a model of a compressible solid. Therefore, given that the classic Mooney-Rivlin and Yeoh models characterize incompressible solids, an additional incompressibility condition was added for MGM $I_{3}=1$, where $I_{3}=\lambda_{1}{ }^{2} \lambda_{2}{ }^{2} \lambda_{3}{ }^{2}$ is the third invariant of the Cauchy-Green strain measure.

Results of identification of parameters of MG-model of type II (under condition of incompressibility) by diagrams of tension-compression of Yeoh solid are shown bellow in table 1 and fig 3:

Table 1. Parameters of MGM for Yeoh solid.

\begin{tabular}{|c|c|c|c|}
\hline MGM parameters & $\boldsymbol{k} \boldsymbol{a} \cdot \mathbf{M P a}$ & $\boldsymbol{k} \boldsymbol{} \cdot \mathbf{M P a}$ & $\boldsymbol{k} \boldsymbol{d} \cdot \mathbf{M P a}$ \\
\hline Uniaxial stretch & 2.14 & -1.73 & 1.82 \\
\hline Equibiaxial stretch & -0.58 & 5.91 & -8.02 \\
\hline
\end{tabular}


It should be noted that the parameters determined by the same stretch diagram (uniaxial stretch-compression of Yeoh material), but at different limits of elongation ratio, differ. Narrowing the interval naturally improves the approximation quality of the MG model.

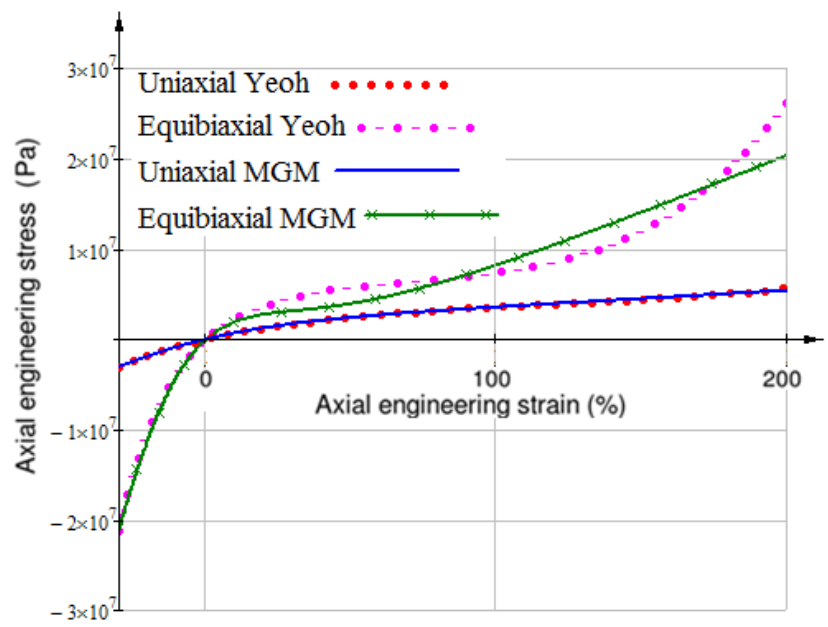

Fig. 3. Stretch diagrams for Yeoh solid and MGM type II.

Results of identification of parameters of MG-model of type II (under condition of incompressibility) by diagrams of tension-compression of Mooney-Rivlin solid are shown bellow in table 2 and fig 4 :

Table 2. Parameters of MGM for Mooney-Rivlin solid.

\begin{tabular}{|c|c|c|c|}
\hline MGM parameters & $\boldsymbol{k} \boldsymbol{a} \cdot \mathbf{M P a}$ & $\boldsymbol{k} \boldsymbol{} \cdot \mathbf{M P a}$ & $\boldsymbol{k} \boldsymbol{d} \cdot \mathbf{M P a}$ \\
\hline Uniaxial stretch & 2.28 & -1.75 & 1.99 \\
\hline Equibiaxial stretch & 0.65 & 4.98 & -7.42 \\
\hline
\end{tabular}

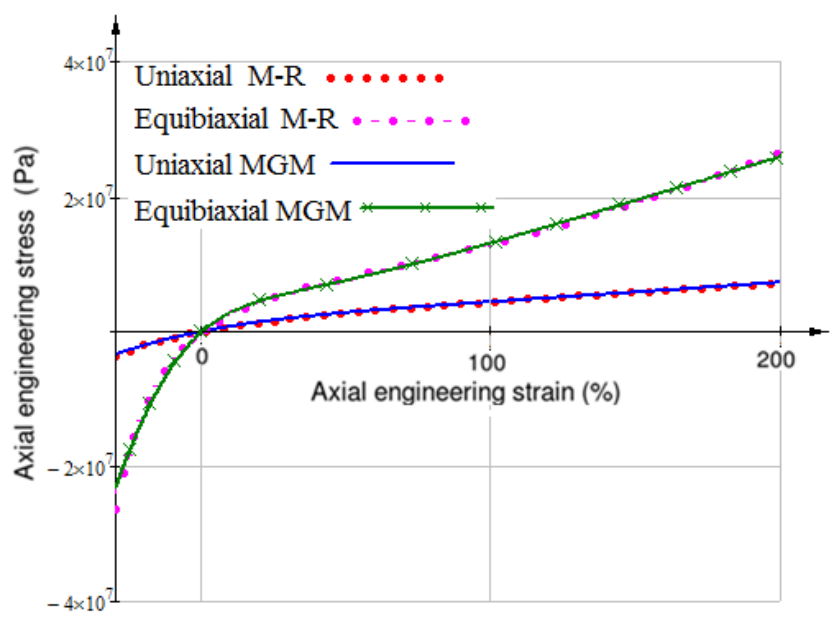

Fig. 4. Stretch diagrams for Mooney-Rivlin solid and MGM type II. 


\section{Conclusion}

The behavior of materials with MGM specific strain energy density generally reflects the properties characteristic of a wide class of nonlinear elastic materials. The specific strain energy density (at least in a certain range of deformations) "a priori" has properties that allow it to be used in nonlinear elastic theory. The source of such certainty is that the method of mechanical-geometric modeling is based on physically consistent assumptions about the geometric and mechanical characteristics of its bonds and structure. A 3D model automatically inherits all the "good" properties of its constituent elements.

The slightly more linear behavior of the mechanical-geometric models compared to the Mooney-Rivlin and Yeoh models can be explained by changing the geometry of the models at large deformations. Descriptions of the substantially nonlinear behavior of a material using a mechanical-geometric model can be achieved by introducing variable mechanical parameters into the model - the nonlinear stiffnesses of the model's bonds. It was shown that it is possible to model elastomeric nonlinear media using this approach.

In general, it is worth noting the wide possibilities of the method of mechanicalgeometric modeling to describe various types of solids. It is possible to model anisotropic solids, which is carried out both by selecting the initial geometry of the model and by choosing different (not identical each other) mechanical characteristics of the bonds. The functions of specific strain energy density thus constructed can describe solids with complex anisotropy. The description of media with viscoelastic $[3,11]$ or elastic-plastic or other properties is also possible within the framework of a mechanical-geometric approach. Thus, in [12], MGM describing viscoelastic media with effects characteristic of such media (relaxation, creep, hysteresis loop) were shown.

\section{References}

1. J. Nasehi Tehrani, J. Wang, Annu Int Conf IEEE Eng Med Biol Soc. November, 7897-7900 (2015) doi: 10.1109/EMBC.2015.7320223

2. C.O. Horgan, M.G. Smayda, Mech. Mater. 51, 43-52 (2012)

3. D. Garcia-Gonzalez, M. Hossain, Int. J. of Solids and Structures 208-209, 119-132 (2021)

4. J. Liang, L., Li, X. Niu, Z. Yu, Q. Pei, Nat. Photonics 7(10), 817-824 (2013)

5. I-Shih Liu, Continuum Mechanics and Thermodynamics 24, 583-590 (2012) DOI: 10.1007/s00161-011-0197-6

6. M.M. Carroll, J. Elast. 103(2), 173-187 (2011)

7. T. Beda, Eur. Polym. J. 50, 97-108 (2014)

8. P. Steinmann, M. Hossain, G. Possart Arch. Appl. Mech. 82(9), 1183-1217 (2012)

9. K. Nomesh, V. Venkateswara Rao, MIT International Journal of Mechanical Engineering 6(1), 43-46 (2016)

10. A. Aidy, M. Hosseini, B.B. Sahari, American J. of Engineering and Applied Sciences 3(1), 232-239 (2010)

11. H. Wang, M. Lei, S. Cai, J. Appl. Phys. 113(21), 213508 (2013)

12. A.D. Azarov, D.A. Azarov, Proceedings of the 2015 Int Conf on Physics, Mechanics of New Materials and Their Applications, devoted to the 100th Anniversary of the Southern Federal University (Nova Science Publishers, New York, 2016) 
13. J.A.S.C. Jayasinghe, K. Wijesundara, R.R.M.S.K. Ranathunga, P. Gamage, W.A.A.S. Premarathna, Lecture Notes in Civil Eng. 94, 517-531 (2021) DOI https://doi.org/10.1007/978-981-15-7222-7_42

14. N. Kumar, A. DasGupta, Int. J. Non Linear Mech. 57, 130-139 (2013)

15. T. Gernay, A. Millard, J.M. Franssen, Int. J. Solids Struct. 50(22), 3659-3673 (2013)

16. A.B. Albrecht, K. Ravi-Chandar, J. Mech. Phys. Solids 64, 377-395 (2013) 